\title{
Interactive remote interviews during emergency remote teaching
}

\author{
Christian D. Solorio $\odot$, Elizabeth Gire, and David Roundy॰ \\ Department of Physics, Oregon State University, Corvallis, Oregon 97331, USA
}

(Received 28 October 2020; accepted 5 August 2021; published 3 September 2021)

\begin{abstract}
The COVID-19 pandemic has made conducting in-person research a health risk for interviewers and participants. Near the start of the pandemic, many universities pivoted to emergency remote teaching where courses were delivered remotely in observance of safety guidelines. The safety guidelines also necessitated that research be done remotely. We designed a remote interview protocol for a computational physics based qualitative interview. Using Zoom's features, we created an interview that allowed participants many modes of interacting with the interviewer and the interview task materials. We present the interview methods and evaluate the utility of remote interviews. We then generalize the experiences from designing and conducting remote interviews for contexts outside of computational physics and describe how task design choices were influenced by technology. We find that conducting interactive remote interviews can be an effective method for physics education researchers even outside of the pandemic.
\end{abstract}

DOI: 10.1103/PhysRevPhysEducRes.17.020114

\section{INTRODUCTION}

The abrupt transition to emergency remote teaching (ERT) due to the COVID-19 pandemic changed not only how we teach, but also how we were able to conduct physics education research. In order to continue research during the pandemic, we developed an interview protocol that was designed to be delivered remotely. The methods by which we conducted the interview allowed the participants to interact with interview task materials beyond verbal communication while maintaining the health and safety of researchers and participants. The features that Zoom and other popular video conferencing software, such as Microsoft Teams, Skype, and Google meet, offer can allow for interactive remote interview tasks, but this has not yet been well explored in the literature. The utility and application of features such as screen sharing, screen annotation, and remote computer control will be discussed in the context of a set of qualitative interviews conducted during May 2020 (relatively early on during the pandemic in the United States). First, we will describe the methods of the remote interviews, the types of interactions participants had with the interviewer and interview task materials, and the efficacy of our set of remote interviews. We will then present some general applications of the features that video conferencing software offers and how those features inform interview task design choices. We will then discuss the

Published by the American Physical Society under the terms of the Creative Commons Attribution 4.0 International license. Further distribution of this work must maintain attribution to the author(s) and the published article's title, journal citation, and DOI. limitations to the generalizability of the use of Zoom's features as well as the ethical considerations one should make when conducting research during a pandemic.

\section{LITERATURE REVIEW}

The development of internet communication has allowed researchers to explore new modes of gathering qualitative data. While the use of remote interviews conducted via video conferencing software such as Skype, Zoom, Google Meet, etc. has not been widely explored in the physics education research context, it has been somewhat explored in the wider qualitative methods literature. Video conferencing software can emulate an in-person interview while addressing some of the scheduling, geographic, and funding constraints of conducting an interview in person $[1,2]$. Several researchers have found that conducting interviews via video conferencing software such as Skype or Zoom can be effective in collecting rich qualitative data [1,3-5], suggesting that remote interviews can be a valuable tool for data collection.

The pandemic has constrained the opportunity to conduct in-person interviews. Understanding how remote interviews compare to in-person interviews is essential for evaluating the utility of remote interviews. This has been explored in the literature. In a comparative study between in-person, Skype, and telephone interviews, Johnson et al. found that remote interviews produced less word-dense transcripts and fewer field notes than in-person interviews, but the remote nature had no significant effect on substantive coding or length of the interviews [6]. They caution researchers that the affordances of remote interviews may come at the cost of richness of the data [6]. Deakin and Wakefield [4] and Sedgewick and Spiers [3] 
found no significant differences between in-person and remote interviews. While remote interviews may not produce data as rich as in-person interviews do, remote interviews are still a viable tool for collecting qualitative data and may have certain advantages over in-person interviews (such as eliminating travel costs and ease of scheduling).

Interviewing participants remotely does introduce some challenges that are not as common in in-person interviews. Participants may face initial technical difficulties joining the call [5], some may have anxiety or unease seeing oneself on their computer screen throughout the interview [4], and there may be environmental distractions to the participant and/or interviewer [4,7]. While technical difficulties are not uncommon to remote interviews, the shared problem-solving experience of troubleshooting technical difficulties can help researchers and participants establish a positive rapport [5]. Despite technological difficulties, participants still have an overall positive experience with remote interviews and researchers can collect usable data from them [1]. The challenges do not outweigh the utility of remote interviews.

Establishing a positive rapport with participants is an essential part of collecting qualitative data [8]. Generally, rapport between researcher and participant is established in person, especially when recruiting participants and at the beginning of an interview. Concerns about building rapport through remote communications have been brought forward [9], however Weller found that building and maintaining rapport during remote interviews was not necessarily more difficult than doing so during in-person interviews [10]. Deakin and Wakefield found that establishing rapport for a remote interview was sometimes quicker than establishing rapport for in-person interviews (depending on the personality of the interview participant), and that remote rapport building is facilitated by the process of exchanging emails or messages prior to the interview [4]. Having visual communication during remote interviews that allow the researcher and participant to read body language and expressions was found to be helpful in establishing rapport $[1,3,5]$. The ability for the researcher and participant to see each other's environments can also help to establish rapport and trust as well, and it possibly facilitates a more personal connection during the interview $[1,7]$. Building rapport is not significantly more difficult in the remote setting, and remote interviews with video help the rapport building process.

\section{REMOTE INTERVIEW CASE STUDY}

We will give instructional and research context for the interviews. We will then describe the remote interview protocol and the types of interactions that participants had with the interviewer and the interview task materials during the remote interview. Finally, we will give an evaluation of the efficacy of remote interviews compared to in-person interviews and a description of the affordances and challenges of remote interviews.

\section{A. Interview context}

\section{Instructional context}

Junior-level physics students at Oregon State University take a sequence of 5-week long intensive courses that meet seven hours per week called Paradigms courses [11]. During the Winter 2020 academic term, most of these students were concurrently enrolled in the introductory spins-first quantum mechanics Paradigms course and a computational physics lab course. Both of these courses were conducted in-person before Oregon State University moved to ERT. During the Spring 2020 term, due to the pandemic, students took all of their courses remotely. The Paradigms courses and the computational physics lab course met synchronously via Zoom.

In the computational physic lab course, students practiced pair programming - a software development technique shown to improve code quality and educational outcomes [12,13]. In pair programming, one student controls the mouse and keyboard to write code (the driver) and the partner gives feedback and suggestions on the code that was written and provides a road map of what needs to be done next (the navigator). The pairs worked in a computer lab writing code in Python and would switch roles approximately every half hour during an 80-min course and partners would change each class session. During ERT, students in the lab course used Zoom's remote computer control feature to control a laptop in a classroom operated by the instructor.

\section{Research context}

Instructors of the quantum Paradigms course have observed that students have difficulties in finding and expressing the connections between discrete and continuous quantum systems, particularly in the context of inner products. Computation offers an opportunity for students to coordinate ideas about continuous and discrete quantum inner products because numerically calculated inner products, even for continuous wave functions, require discrete summation. This motivated us to explore the ways that computation may support students' understanding of discrete and quantum systems.

Starting in February 2020 (before ERT had begun) we collected observational data of six students in the computational physics lab course for a research project. We had anticipated being able to conduct a set of in-person, semistructured interviews to complement the in-class observational data near the end of the academic term. Oregon State University, however, moved to ERT at the end of March 2020 (before we had developed an in-person interview protocol). In order to maintain the cohesive, indepth nature of the study, we developed an interview 
protocol that was meant to be delivered remotely while allowing for many modes of interaction between the interviewer, participant, and interview task materials.

\section{B. Interview description}

Because pair programming has been shown to decrease the amount of time needed to produce functioning code [14], we felt it would be too difficult and possibly stressful for participants to write functioning code without a partner in a 60-minute interview. In order to maintain the computational nature of the interview, we developed a protocol that combined a pseudo-code task with a code manipulation task.

The interview protocol was not designed to follow a pair programming model in order to minimize the influence that the interviewer would have on the participant during the interview tasks. While our interviews did not incorporate pair programming, we expected participants' previous experience doing pair programming to be beneficial. For example, because pair programming improves the communication between students working on code [15], we anticipated participants being comfortable communicating technical details of code as well as verbalizing their thoughts and actions while doing a computational task.

We used Zoom's remote computer control feature so that the participant could manipulate documents, code, windows, etc. in a coding environment that they were familiar with from their computational lab course. This design choice allowed participants agency in how they would interact with the interview task materials (as would also be common for an in-person interview) and minimized the influence that the interviewer had on the participant's actions and explanations. Additionally, the familiarity with the text editor that was used to display and execute the code meant that participants did not need to learn a new coding environment without having to install any software on their computer.

Each interview was conducted one-on-one with the interviewer and one of the six participants. The interviews were semi-structured in nature, approximately one hour long, and were conducted remotely via Zoom. The webcam, audio, and interviewer's computer screen were recorded using Zoom's built-in recording feature. The full interview protocol can be found in the Appendix.

\section{Pseudo-code}

A common coding practice is writing pseudo-code, which is an informal description of code or piece of code that does not follow the syntax requirements of a coding language. This is not a practice that students were directed to use in their computational physics lab course, but we decided it would be a useful interview task because it allowed us to probe the participants' translation of physical concepts and mathematical operations into an algorithmic form. Additionally, pseudo-code does not require remembering or debugging programming language syntax, lessening the cognitive load on the participants.

Participants were given a description and example of pseudo-code before starting the activity. They were asked to write pseudo-code for the inner product between two spin-1 (discrete) states and then for the inner product between a wave function and a generic energy eigenstate. The interviewer shared their screen using Zoom's screen sharing feature and displayed a document containing the written prompts of the questions. The participants were granted remote control of the interviewer's computer so that they could type their pseudo-code directly into the document containing the interview task prompts.

\section{Code manipulation}

Immediately following the pseudo-code task was a code manipulation task where participants were presented with a pre-written Python code (see Appendix). The pre-written code approximates a wave function of a particle in a box by expanding the wave function up to the first 10 terms in an energy eigenstate expansion. The code generates two plots -one that shows the inner products of the wave function and an energy eigenstate $\left(c_{n}\right)$ versus the energy level $(n)$, and another that shows both the wave function and the approximation of the wave function as a function of position. The participants had produced similar code in their computational physics lab course and were familiar with the context needed to understand the prewritten code and its output.

The prewritten code was built to produce physically incorrect visualizations. Weatherford and Chabay have previously used code that runs but produces inaccurate visualizations [which they call minimal working programs, or (MWPs)] in instructional tasks [16]. Instead of omitting lines of code as Weatherford and Chabay did for their MWPs, we set a parameter in the code to a value that would produce unphysical visualizations. In the code, the leftRiemann sum rectangle width parameter, $d x$ (used in calculating the inner product $c_{n}$ ) was set to be one-quarter of the box length. This produces atypical behavior in the plots, e.g., the $c_{n}$ vs $n$ plot showed periodic behavior and the approximation of the wave function produced an approximation with more extrema than the wave function. This bug was included to elicit participants' reasoning about the importance of the $d x$ parameter in a continuous inner product.

The code was opened using the same text editor that participants had used in their computational physics lab course. The interviewer's screen was shared, and participants were given remote control of the interviewer's computer. Participants were prompted to execute the code, describe what the code was doing, what they thought about the output, and to modify the code to produce physically accurate plots. 


\section{Remote interview interactions}

In-person interviews tend to have a high level of interaction between the interviewer, interview participant, and materials used for the interview task. Often, interview tasks make use of whiteboards and markers, pre-printed materials such as graphs and equations, three-dimensional surfaces, etc. Many remote qualitative interviews have focused just on participants' verbal responses. The remote interviews we conducted, however, were designed to allow participants many ways of expressing their thoughts beyond just verbal communication in order to gather indepth data. The different modes of interaction between the interviewer, participant, and interview task materials during this set of remote interviews will be described in this section.

Webcam and audio: Participants were asked to use both their microphone and their webcam (if they had access to one) throughout the interview. This allowed for the basic communication that the interviewer and the participant would have in an in-person interview. Both the interviewer and participant could see each other's facial expressions, gestures, and some body language (dependent on the framing of one's webcam) as well as hear each other. One participant $\mathrm{F}$ did not have access to a webcam, but they were still able to do the interview tasks and communicate their understanding of the physics. The interview with this participant produced data similar to other interviews (e.g., there was audio data, screen recording data, the interviewer and participant were able to influence the flow of the interview), although it lacked the context of facial expressions and gestures.

Screen annotation: Zoom offers a screen annotation that, unlike a virtual whiteboard, allows participants to circle snippets of code, add features to a plot, draw lines connecting code to plots, etc., directly on a shared screen. Screen annotation was used throughout the Paradigms courses during ERT, but participants were not prompted to use this feature during the interview. Two of the six participants used screen annotation to supplement their explanations of the prewritten code provided in the code manipulation task. Participant $\mathrm{F}$ used screen annotation (seen in Fig. 1) to explain the role of $d x$ in integration by drawing the rectangles for a Riemann sum, stating "I think of integrals as rectangles..." Participant A used the annotation feature to explain the contributions of certain eigenstates in the approximation of the wave function, drawing lines on the wave function and approximation versus position plot after they had trouble describing this in words.

Remote computer control: Using Zoom's remote computer control feature allowed for several modes of interaction with the interview task materials, which are described below.

- Pseudo-code: Each participant was prompted to type their pseudo-code into a word document on the
8. Figure 2

$\square \quad \times$

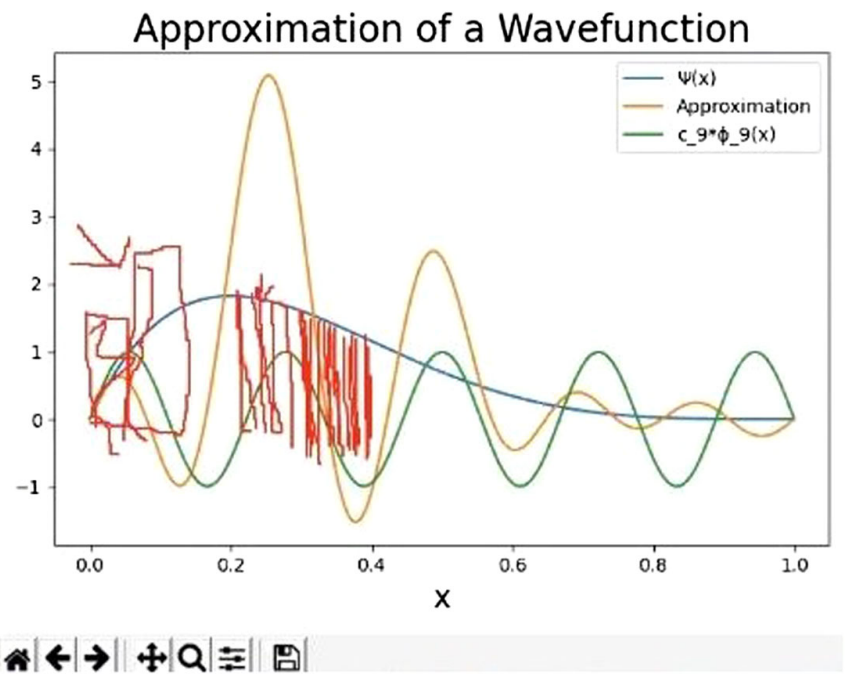

FIG. 1. Participant F used screen annotation to draw on a plot with probability density on the vertical axis and position on the horizontal axis to enhance their explanation of the role of the size of $d x$ in a Riemann sum. The plot shows a wave function (blue) and the approximation of the wave function (blue), the ninth energy-eigenstate term of the approximation (green), and the participants Riemann sum annotation (red).

interviewer's computer via Zoom's remote computer control feature. The word document allowed participants to organize and modify their pseudo-code in a way that was visible to both the participant and interviewer without much lag. Most participants' pseudo-code resembled Python code, using the appropriate syntax for mathematical operations and new lines in the word document for each part of their pseudo-code. One participant, though, generated pseudo-code that used almost exclusively words instead of Python syntax for addition, multiplication, loops, etc.

- Code manipulation: The operating system, programming language, and code editing program (Atom) used in the remote interview were identical to what students used in the remote computational lab course. Participants were given the option to manipulate and execute the code themselves, or to ask the interviewer to do it if there was significant lag in the Zoom connection. Each of the six participants were able to modify and execute the code themselves using Zoom's remote computer control function. This allowed participants to modify the existing code to explore their understanding of the role of $d x$.

- Window arrangement: Python's matplotlib package generates interactive plots in individual windows which can be moved and resized. Three participants rearranged windows containing similar plots to make direct comparisons between them. As shown in Fig. 2, 


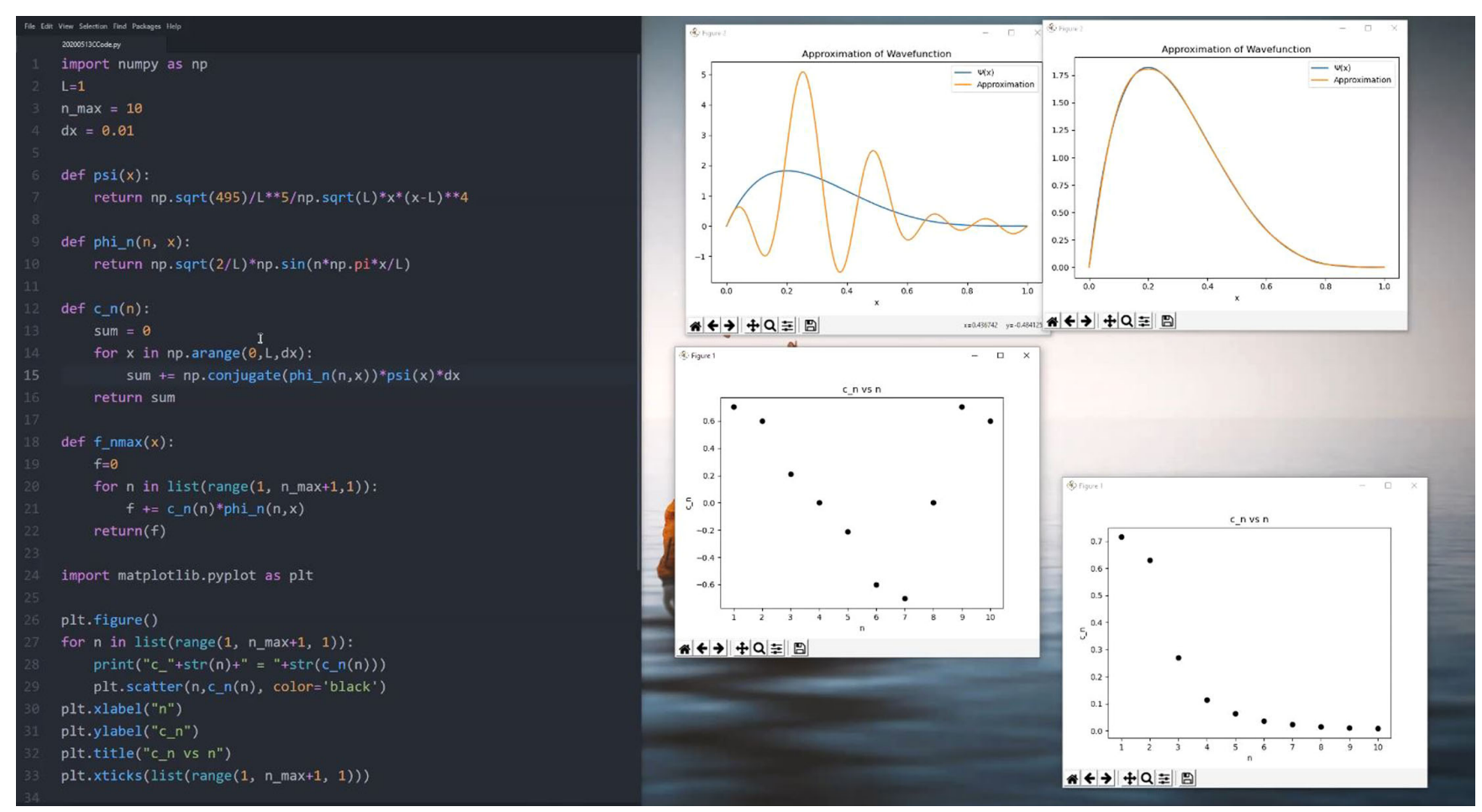

FIG. 2. An image of the screen of the interviewer's desktop where participant $\mathrm{C}$ arranged the windows to make comparisons between plots. On the left-hand side of the image is the code participants used during the code manipulation task. The top plots display the wave function (blue) and wave function approximation (orange) with probability density on the vertical axis and position on the horizontal axis. The bottom plots display $c_{n}$ on the vertical axis and $n$ on the horizontal axis. The plots on the left have $d x$ set to 0.25 (the initial value) and the plots on the right have $d x$ set to 0.01 .

Participant $\mathrm{C}$ intentionally arranged the windows such that the wave function and wave function approximation plots are next to each other and the corresponding $c_{n}$ plots are directly beneath. He then explained, "So, your $d x$ changed your integration of the inner product" when comparing the two $c_{n}$ plots. The agency to spatially organize information facilitated participants' explanations.

- Plot manipulation: The plots in Python's matplotlib are interactive, allowing the user to zoom in and out of and to pan around features on the plot. Participant G used the interactive nature of the plots to zoom into the lines on the wave function and approximation versus position plot to evaluate the accuracy of the approximation. This mode of interaction with the interview task materials allowed the participant to explore specific features of the plot which guided them in how they chose to manipulate the code.

\section{Efficacy of interviews}

In order to evaluate the efficacy of the remote interviews compared to in-person interviews, we apply a framework of interview considerations that examines the form and outcomes of the remote interviews. In particular, we address the quality of communication between the interviewer and interview participant as well as the infrastructure of the remote interview methods.

\section{Verbal information exchange}

The remote interview protocol was designed to be semistructured, which requires a continuous exchange of verbal information between the interviewer and interview participant. As in a face-to-face interview, the remote interviews did not interfere with the interviewer's ability to adjust, reorder, change, add, or forgo questions in response to the interview's flow. The interview participants were able to respond to the interviewer's questions, ask clarifying questions, and influence the flow of the interview. While there is the possibility of equipment failure that would compromise verbal exchanges during a remote interview, we did not encounter any such failures (and we had contingency plans in case such a failure had occurred). We find that verbal exchanges over a remote medium were very similar to verbal exchanges in person.

\section{Multimode communication}

In addition to verbal exchanges, in-person qualitative interviews commonly use multiple modes of nonverbal communication that include body language, facial expressions, gestures, sketches, writing, etc., to help participants 
fully express their thoughts. The remote interviews we conducted facilitated the use of multiple modes of nonverbal communication. Participants were able to type in word documents (six of six participants), edit Python code (six of six participants), gesture or convey body language via webcam (five of six participants), arrange windows to organize or compare code output (three of six participants), annotate the interviewer's screen (two of six participants), manipulate plots (one of six), and write math on paper that they displayed via their webcam (one of six participants).

\section{Fluency}

In addition to having multiple modes of communication throughout an interview, the ease of using and switching between the modes of communication, or fluency, plays an important role in an interview. In-person interviews accomplish this by allowing participants to support their explanations with familiar tools such as verbal explanations, gestures, whiteboards and markers, etc. We found our set of remote interviews to accomplish fluency with the possible modes of communication participants could use.

Each of the participants were very familiar with Zoom and its features because of the six weeks of using it for courses during ERT. Little time had to be devoted to explaining how to operate Zoom to complete interview tasks (participants were familiar with typing in Word documents, operating a remotely controlled computer, coding in a familiar environment, and using screen annotation). Switching between verbal and non-verbal modes communication was relatively fluent for most participants. The participants' familiarity with Zoom allowed participants agency in how they chose to express their thoughts-they could utilize screen annotation to draw, type in word documents and code editors, manipulate windows to organize information, make hand gestures, etc., and switch between modes of communication with relative ease. Participants did not express any hesitancy in using and switching between any of the outlined modes of communication.

Some aspects of communication, however, were limited. Verbally expressing math, writing math with a mouse, or typing math is more difficult than using a whiteboard and marker, for example. In one interview, participant $\mathrm{C}$ opted to write math on paper and then show their work over the webcam instead of using a virtual whiteboard or screen annotation. In another interview, participant $B$ experienced a noticeable lag between their typing on the interviewer's computer via remote computer control and their verbalization of what they were doing due to internet bandwidth limitations. While this was not a major constraint on their ability to communicate, but it did somewhat affect the flow of the interview.

In general, participants of the remote interviews were able to express their thoughts through a variety of modes of communication with varying degrees of ease and were able to switch between modes of communication without major interruptions to the flow of the interview. Remote interviews may be less fluent than in-person interviews, but not to an extent that participants are overly constrained in how they express their thoughts.

\section{Data capture}

In-person data capture may involve multiple cameras and microphones to fully document what the participant said and did throughout the interview. Setting up data capture for the remote interviews was relatively simple since Zoom's recording settings can be configured ahead of time. For each interview, we were able to collect audio data and webcam data (when possible) from the interviewer and participant, as well as the interviewer's shared screen. The recording was then saved directly on the interviewer's computer, avoiding any need to transfer data from a camera and microphone to a computer. While remote data capture can be simple, there are some limitations compared to inperson data capture. The fidelity of the recorded data partially depends on the quality of the participant's webcam and microphone, resulting in some variety of video and audio quality between interviews. Each of the participants were audible and each participant with a webcam was visible, but some were easier to hear or see depending on the participant's equipment. The field of view of the webcam was another limitation of the remote interview. The interviewer was only able to see the participant's upper body which made reading body language more difficult. The limited field of view of the webcam also meant that the interviewer could not see what a participant was writing or sketching on paper in real time.

There are several data capture failure modes introduced in the remote interview setting as well. Equipment like webcams, microphones, and computers can fail, programs may crash, internet connection can be slow or lost, and power outages can occur. Budgeting time for the possibility of troubleshooting did constrain the amount of time that could be devoted to interview tasks (but may also help form rapport between the interviewer and participant [5]). We did not encounter any significant technological difficulties during our interviews, but we had pre-established contingency plans to navigate the common issues if they came up. The contingency plans may affect the uniformity of data, however (for example, one out of the six participants did not have a webcam). We were able to successfully capture the essential data (audio and computer screen footage) in each remote interview that was clear and able to be used in analysis.

The data we were able to collect were rich (as evidenced by many modes of interaction participants had with the interviewer and interview task materials captured via video, audio, and screen sharing recordings) despite the interviews being conducted remotely. The data we were able to capture were comparable to the data we would want to collect during an in-person interview (audio, video of the 
participant's facial expressions and body language, and video of the participant doing an interview task). This agrees with several other studies that find that remote qualitative interviews can produce rich data [1,3-5].

\section{Privacy and safety}

In-person interviews are generally conducted in a space that maintains the privacy and safety of the interview participants. The main motivation for us to conduct interviews remotely was to maintain the interviewer's and participant's safety from the transmission of COVID-19. Both the interviewer and participants were in their respective homes while conducting the interview. For some participants, being in their own space may be less stressful than being in an unfamiliar space like a research lab, allowing the participant and researcher to build rapport and trust [1,7]. During the pandemic where people are more restricted in the spaces that they can occupy, this may not be true, however.

While there are benefits to interviewers and participants being in their own space for rapport and trust building [1,7], being at home can offer distractions that a research lab interview space would not. For example, the interviewer's cat walked across their keyboard during an interview which distracted a participant while they were expressing their thoughts. In another interview, a participant was working in a kitchen space with someone occasionally walking through which occasionally distracted them. Distractions during a remote interview may be exacerbated by the pandemic when people (especially students sharing a dorm room, house, or apartment) have to share a space.

For our set of remote interviews, participants were not given any restrictions on where they could be during the interview. Four of the six participants were in a private room while being interviewed, one participant was in a kitchen space without anyone else present, and one participant was in a kitchen space with another person occasionally walking through. In addition to participants choosing their interview location, participants were able to select preferred times for their interview between $9 \mathrm{AM}$ and $8 \mathrm{PM}$ over an eight-day period. While each of the remote interviews we conducted took place between $10 \mathrm{AM}$ and $5 \mathrm{PM}$ on a weekday, participants were given the option to choose interview times at night when an in-person interview may be less safe or convenient. None of the participants appeared or sounded uncomfortable or unsafe during their interview.

Our set of remote interviews relied heavily on remote computer control. One of the motivations for this was computer privacy. By having participants do the interview tasks on the interviewer's computer via remote computer control, the participants had a higher degree of personal privacy. Had participants shared their screen, they may have accidentally displayed their files, desktop notifications, emails, messages, etc. The interviewer was able to configure their computer beforehand to avoid this issue while sharing their screen.

The remote interviews we conducted maintained the privacy and safety of participants to the best extent possible by offering flexibility in the interview environment and time. None of the participants appeared or sounded concerned about their personal privacy during their interview. While remote interviews may be more prone to distractions, especially while stay-at-home orders are in effect, than inperson interviews, remote interviews did not compromise on the safety or privacy of participants.

\section{Accessibility}

Accessible interviews allow researchers to be more equitable in their research and reach a wider pool of possible participants. One major advantage of the remote interview compared to an in-person interview is that the interviewer and participant are not limited to any particular geographic location which can alleviate the need of travel and the possibility of having to navigate a campus that is lacking in accessibility. Additionally, many video conferencing software platforms offer assistive technology capabilities such as live captioning and screen readers. In the case of screen sharing and remote desktop control, the interviewer can install screen reader software on their machine for participants who need such accommodations. While none of the remote interview participants requested such accommodations, we would have been able to provide them as we would have during an in-person interview.

\section{GENERAL APPLICATIONS TO REMOTE INTERVIEWS}

While the computational context of the interview tasks made creating an interactive remote interview protocol easier, the methods used in this case study are generalizable to other contexts. By utilizing more of the video conferencing software's features, interviewers can conduct a wide variety of interview tasks and collect the same kinds of data (audio, video, gestural, etc.) that are comparable to what an in-person interview would offer. Here, we will discuss how video conferencing features can influence logistical choices and task design choices for a remote interview.

\section{A. Video conferencing software}

One of the first design choices we made for our set of interviews was a video conferencing software to use to conduct the interviews. We chose to use Zoom because it was the video conferencing software that participants were most familiar with because of its use for remote course instruction. Most of the features that Zoom offers can be reproduced on other popular platforms such as Microsoft Teams, Skype, and Google Meet (with the Chrome Remote Desktop extension for the Chrome web browser). Zoom, however, is the only software that has a screen annotation 
feature, allowing for one to draw directly onto a shared screen instead of a virtual whiteboard. This feature was used by two participants to supplement their explanations, but screen annotation was not necessary for conducting our interactive remote interviews. The degree of customization of the features on each video conferencing software platform listed varies, but our research methods could be reproduced on any of these platforms.

Interview task design choices are constrained to the capabilities of video conferencing software. For the purposes of our interviews, computational tasks transfer relatively easily to the remote platform because remote interviews require a computer. Activities that require the physical manipulation of objects such as card sorting tasks [17] and 3D surface exploration [18], however, are more difficult to conduct in the remote setting. Adapting such tasks to the remote setting while maintaining the key aspects of the task can be possible by emulating the physical objects in a virtual environment that allows for the objects to be manipulated. Alternatively, the physical objects could be mailed to the participant before the interview.

\section{B. Webcam and audio data}

By utilizing webcam and audio feeds, remote interviews can capture some of the essential elements of an in-person interview as discussed in Sec. III D. Having an audio connection to a participant allows for verbal communication and is generally necessary for qualitative interviews, but the need for a video feed may be more flexible. A video connection grants the interviewer access to data based on facial expressions, gestures, and body language during the interview. A video connection can also be beneficial in establishing a rapport with participants $[1,3,5]$.

During the design of the interview tasks that we used in the set of interviews we conducted, we decided that having video data of the participant was not essential for answering our research questions. The essential pieces of data were the audio and the screen recording because the interview was focused on participants' communication of computational and physical ideas regarding discrete and continuous quantum systems. Depending on the nature of the research questions one is attempting to answer, webcam video may be a required form of data to collect. The need for video data can allow the interviewer to ask a wider breadth of research questions (such as those with a kinesthetic component) but will create more selection criteria for participants.

\section{Screen sharing}

Many in-person interview tasks make use of visual information such as equations, images, plots, etc. Sharing that type of information over the webcam may not be sufficient, as the fidelity of the image depends on the quality of the interviewer's webcam and the interviewer and participant's internet connection. Sending files to a participant via email or a video conferencing software's chat is not always an elegant solution as it can require multiple messages depending on how many files need to be shared, and it can become difficult for the interview participant to keep track of multiple files. There are also times where the sequencing of visual information is important for an interview task. Allowing the interviewer to easily introduce material when they find it to be appropriate via screensharing can allow for interview tasks that depend on the sequencing of the introduction of visual information. Screen sharing allows both the interviewer and participant to see visual information in a high quality and the interviewer maintains control of when information is brought into the interview.

\section{Remote computer control}

Screen sharing may not always be sufficient for the interview task. Certain interview tasks may require software that participants do not have on their computer. For example, in our interviews, participants manipulated code and plots from a Python script, but some participants did not have Python and the appropriate packages installed on their computer. Other interview tasks may require licensed software, such as Mathematica, to produce plots that a participant can manipulate and interact with during the interview. By utilizing remote computer control, participants do not need to purchase any licenses or install any software on their computer to participate in the interview task.

Remote desktop control also avoids any difficulties associated with the participant's computer (such as incompatibilities with the participant's operating system, the ability for the participant's computer to reliably run multiple programs simultaneously, and out-of-date software), giving it an advantage over having a participant do an interview task on their computer while sharing their screen.

The use of remote computer control allows for interviewers to use whatever software they find suits their interview task while avoiding technical and logistical issues, allows for the participant to interact with the interview task material in a direct manner, and maintains the privacy of the participant's personal files, emails, messages, notifications, etc. making it a useful tool for certain interview tasks.

\section{E. Screen annotation and virtual whiteboards}

Being able to interact with interview task materials allows for participants to better express their thoughts while doing a task. Screen annotation, a feature that is unique to Zoom, allows users to draw directly on a shared screen. Screen annotation allows for participants to add to images in ways that they would be able to add to images on paper during an in-person interview. For example, one of the participants in our set of remote interviews used screen annotation to draw what the curve of the approximation of 
the wave function would look like if the contribution of one term in the energy eigenstate expansion was subtracted.

While screen annotation is unique to Zoom, many other video conferencing software offer a virtual whiteboard feature that allows users to write, draw, insert text, etc. The virtual whiteboards do not allow a user to draw directly onto a shared screen, however. Including prompts in the interview protocol for participants to use screen annotation or a virtual whiteboard gives participants more ways to express their thoughts and display their understanding during an interview.

\section{F. Contingency plans}

In an in-person interview in a research lab space, the interviewer has control over the environment, tools, and equipment necessary for data capture. Remote interviews do not offer this same kind of control as participants use their own computer, webcam, and microphone and they are more free to choose when and where they are to be interviewed. Additionally, the reliance on technology to conduct the interview introduces opportunity for things to go wrong (as outlined in the data capture description of the interviews in Sec. III). Having pre-established contingency plans for common issues can avoid losing interview time. Many technical issues are symptoms of internet bandwidth issues and therefore encouraging participants to use a wired connection or to be located in a spot near their internet source may help in avoiding such issues. Equipment issues may be more difficult to plan for. Deciding what components of the interview data are essential and which parts can be dropped to facilitate the remote connection should be considered during the development of an interview task.

\section{G. Geographic location}

While the participants we conducted interviews with were in or near the same geographic location of the interviewer, the biggest advantage of remote interviews is that they do not require the interviewer and participant to be in the same geographic location. This use-case for remote interviews has been studied before $[1,4,19]$, but remote interviews have not often been used in PER. Remote interviews allow physics education researchers to reach a larger population of participants and to ask questions with a geographical component, such as comparative studies between different universities. This expands the breadth of research questions that physics education researchers can explore and may influence the design choices that one makes when creating an interview task. Additionally, it can allow researchers to save time from setting up interviews, transferring data, commuting to a location and money from traveling and purchasing recording equipment $[4,20]$.

\section{DISCUSSION}

\section{A. Limitations to generalizability}

Because of the ongoing pandemic situation during the remote interviews, the topic of the research, and the context of the research project, there are aspects of the remote interviews we conducted may not be generalizable to all remote interviews with an interview task. We will explain some of the limitations to generalizability that are present in the remote interviews we conducted here.

Because of emergency remote teaching due to the pandemic, participants had become very familiar with Zoom and its features by the time the interviews took place. Very little time during the interview had to be spent explaining how to use the video conferencing software or troubleshooting technological issues. Additionally, two participants used screen annotation to express their thoughts throughout the interview. Their familiarity with Zoom facilitated their use of multiple modes of communication. Outside of a pandemic that necessitates emergency remote teaching, students may not be so familiar with video conferencing software. Asking participants to install and learn a new software may contribute significantly to their cognitive load during the interview. The participants may also require more explanation of how to use the video conferencing software and its features to encourage multiple modes of communication throughout the interview.

The remote interview tasks we developed had a computational context and we conducted the interviews over Zoom, a feature-rich video conferencing software that facilitated the development of an interactive interview protocol. Developing an interview task for a remote interview that does not have a computational context could be more difficult, however. The technology (and the interviewer's and participants' familiarity with it) used to conduct the interview may play more of a limiting factor in how a participant can express their thoughts and understanding.

The interviews we conducted were a part of a larger project that included observations of the participants in their computational physics lab course. The interviewer conducted observations of the six participants for $80 \mathrm{~min}$ twice a week for five weeks. During this time, the interviewer and participants were able to build a strong rapport in-person before the remote interviews took place. As discussed in Sec. II, establishing rapport in the remote setting is not significantly more difficult in the remote setting [10]. Building rapport remotely can be achieved by exchanging emails prior to the interview [4] and utilizing video throughout the interview $[1,3,5]$. The differences between doing a remote interview where rapport had been previously established face-to-face and a remote interview without previous face-to-face requires further investigation. 


\section{B. Ethical considerations}

The COVID-19 pandemic has had a significant effect on the mental health of young adults in the United States $[21,22]$ and worldwide [23-25]. Asking students to be open and vulnerable enough to participate in a remote interview during the spring of 2020 as the pandemic was growing in the United States was a significant request to make. Students were adjusting to transitioning in-person interactions to remote interactions.

The stress and isolation that students were experiencing was considered during the development of the remote interview protocol. We limited the length of the interviews to $1 \mathrm{~h}$ and offered flexibility in scheduling to minimize the "Zoom fatigue" associated with spending many hours a day using video conferencing software for courses and social interaction. We also developed the interview task to be something fairly familiar to participants that also offered insight into their understanding of discrete and continuous quantum mechanics in the computation context that the observations did not offer. The mitigation of stress does not erase the stress, however, and the data collected from interviews may be influenced by this.

Remote interviews require a computer, a microphone, a webcam, and a stable internet connection (with a high bandwidth if streaming video, audio, and a shared screen is required). Researchers should decide on what data is necessary to collect to answer their research questions and what equipment will be necessary to gather that data early in the design of the remote interview. Some participants will not have access to all of the necessary equipment, however. Creating a selection criterion based on who owns all of the necessary equipment could bias the research by systematically excluding low-income students. In order to somewhat mitigate this bias in our own research, we decided that a webcam was not a necessary piece of equipment for participants to have.

\section{Conclusions}

Traditionally, remote interviews have been constricted to conversations through webcam and audio, making them less interactive than in-person interviews. Using the features offered in video conferencing software, we were able to collect data similar to what we would be able to collect in an in-person interview. Most video conferencing software can record the interview, making the collection of audio, gestural, facial expression, and screen-capture data possible and perhaps less obtrusive than a camera setup for an inperson interview. Many popular video conferencing software also has screen sharing and remote computer control features which facilitates the development of interactive remote interview protocols. We have shown that using screen sharing, remote computer control, and screen annotation during a remote interview allowed for participants to do interactive interview tasks that generated rich data in our case study. Additionally, we generalized the knowledge we gained from conducting a case study of remote interviews to apply more generally to researchers developing a remote interview protocol.

From the development and conduction of remote interviews, we found that the interactions that participants had with the interviewer and interview task materials were strongly shaped by the features and limitations of the technology being used in the interview. Understanding the features and limitations of the video conferencing software is essential for determining what data one will be able to collect. For example, screen annotation and remote computer control are two Zoom features that allow for many types of remote interactions during a problem-solving interview that a webcam and audio only interview could not accomplish. There are limitations to Zoom as well, such as the virtual whiteboard feature that allows users to draw on a shared space using their mouse or another input device. The strokes that users make though do not appear in real time, which means the interviewer may miss intermediate steps in a participant's drawing. This prompted us to use a word document instead, which limited the participant's ability to structure the pseudo-code. Having an understanding of the video conferencing platform allowed us to create an interactive interview task capable of being done by participants remotely. The technological design choices (such as video conferencing software, the features of the software necessary for the interview task, and any other websites or software being used) inform and constrain the design choices that one makes while developing an interview task.

There are several benefits to conducting interviews remotely. Remote interviews can include interactive tasks that facilitate the collection of rich data. They grant participants more flexibility in when and where they are interviewed, which can impact the comfort level of and rapport with participants. Remote interviews can also help new researchers save money on equipment such as cameras, microphones, and memory cards. Additionally, remote interviews allow researchers to reach a wider pool of participants who are not in the researcher's geographical area. This allows for a broader pool of participants and allows researchers to explore questions that seek to compare and contrast participants at different universities, backgrounds, etc. without adding significant cost to the research project. For these reasons, remote interviewing can be a useful tool for physics education researchers even postpandemic.

\section{ACKNOWLEDGMENTS}

This work was supported by the National Science Foundation (Grant No. DUE-1836604). 


\section{APPENDIX: REMOTE INTERVIEW PROTOCOL}

The interview protocol that the interviewer followed is given below. The text was meant to be read aloud at the appropriate times during the interview. Actions are enclosed in brackets. The interview was designed to be semistructured therefore the interview protocol may not have been strictly followed for each interview.

\section{Introduction}

I will start recording the session. If you consent, please select that option as it appears on your screen. Thank you for meeting with me today. This interview should last no longer than $1 \mathrm{~h}$. Your participation here is voluntary and you may ask for us to stop at any time. If you would be comfortable communicating face-to-face, please turn on your webcam if you have one. I am not so much looking for correct answers as I am looking for your understanding of quantum mechanics and computation. Because I don't want my expressions to influence what you are saying and doing the interview, I will maintain a neutral expression.

\section{Pseudo-code explanation}

In part of this interview, I will ask you to do some pseudo-coding. There's no correct or incorrect way of pseudo-coding, but occasionally I may ask you to explain or clarify something. Pseudo-code is an informal general outline of a program or function, using mostly words. You don't need to worry so much about the exact syntax or names of python functions - as long as you are saying what you are thinking and what you want to happen, I will understand. Here is an example of pseudo-code [open the document "InterviewTaskSheet.docx" and share screen to show the pseudo-code example as shown in Fig. 3, explain the example].

[Ask if the pseudo-code explanation makes sense. If the participant responds yes, continue on. If not, ask what part the participant needs more help understanding.] I will share my screen and grant you remote control of my computer. You will be able to organize your thoughts in this word

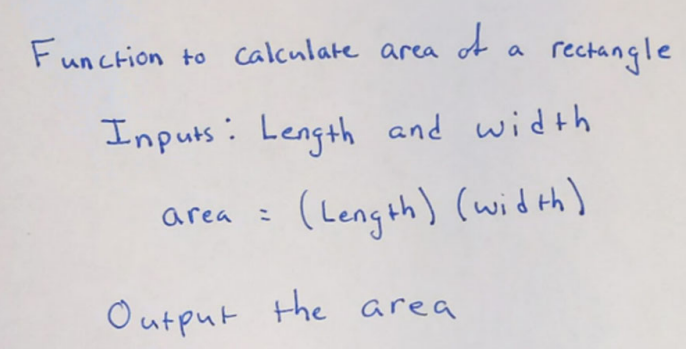

FIG. 3. An example of a pseudo-code for calculating the area of a rectangle. This function takes in two inputs - a length and a width. It multiplies the length by the width and stores that as the area, which the function outputs. document [Share the desktop screen and the participant remote control of the computer].

\section{Pseudo-code (20 min)}

In this part of the interview, I will ask you to generate some pseudo-code.

1. How would you describe in pseudo-code computing the inner product of these two spin-1 states?

$$
\begin{gathered}
|\alpha\rangle=\frac{2}{\sqrt{14}}|-1\rangle+\frac{1}{\sqrt{14}}|0\rangle+\frac{3}{\sqrt{14}}|1\rangle, \\
|\beta\rangle=\frac{1}{\sqrt{2}}|0\rangle-\frac{1}{\sqrt{2}}|1\rangle .
\end{gathered}
$$

(a) If they ask if it is $\alpha$ on $\beta$, or $\beta$ on $\alpha$, etc. tell them that they may choose. If time allows, ask them what difference it would make if they did the inner product the other way.

(b) If they are unsure of how to translate this to code, scaffold as appropriate; e.g., ask them how they would do it algebraically.

(c) If they are unsure of how to work with the spin-1 system, ask them to do the same task with the following spin- $1 / 2$ states.

$$
\begin{gathered}
|a\rangle=\frac{\sqrt{3}}{2}|+\rangle+\frac{1}{2}|-\rangle, \\
|b\rangle=\frac{\sqrt{1}}{\sqrt{2}}|+\rangle+\frac{1}{\sqrt{2}}|-\rangle .
\end{gathered}
$$

2. How would you describe in pseudo-code computing the inner product of the wave function below and an energy eigenstate of a particle in a box ?

(a) If they ask if the inner product is $\psi$ on $\phi$ or $\phi$ on $\psi$, tell them they can choose. If time allows, ask them what would change if they did it the other way.

(b) Time allowing, ask what basis are they working with. If they say energy, ask, "Some would say this is written in the energy basis. What do you think about that?" and vice versa.

$$
\begin{gathered}
\psi(x)=A x(x-L)^{4} . \\
\phi_{n}(x)=\sqrt{\frac{2}{L}} \sin \left(\frac{n \pi}{L} x\right) .
\end{gathered}
$$

(c) If they are using the wrong definition of the inner product, after they finish their pseudo-code, show them the following definition that they have used in the computational lab course. Ask them how having this definition would change 
their pseudo-code.

$$
\langle\phi \mid \psi\rangle=\int \phi(x)^{*} \psi(x) d x
$$

3. What are some of the differences or similarities between calculating the inner product of a spin-1 system, and calculating the inner product for a particle in a box?

(a) If they only list similarities, ask if there are any differences and vice versa.

\section{Code manipulation (20 min)}

In this part of the interview, I am going to show you some code written at about the level of a PH 365 student by sharing my screen. I will run the code to show you a couple plots. I will ask you to interpret the code and output. I will give you remote control of my computer to modify and rerun the code. If there are any issues with the remote control, you are allowed and encouraged to ask me to modify the code as you see fit. I am interested in your understanding of the content, not about correct or incorrect answer. [Open the code "InterviewCode.py" and share screen, giving the participant remote control of the computer.]

Note: Interview questions should be asked in an organic manner when they come up. The order below does not necessarily reflect the order that they will be asked in the interview.

1. Please start off by looking at the code and looking at the output and tell me what you are seeing and thinking of.

2. What do you think about the output of the code?

3. Do you think the code is producing a good approximation?

4. Is there anything you would like to modify about the code?

(a) If they at no point want to change $d x$, ask them what happens when they change $d x$.

5. Why is the original approximation bad?

6. What do you think about the decreasing amplitude as $x$ increases?

7. Why do you think the peaks appear where they do?

8. Going back to a question I asked earlier, what are some of the similarities and/or differences of an inner product for a spin-1 state and an inner product of a particle in a box?

\section{Interview code and plots}

The Python script InterviewCode.py is used for the code manipulation task. The plots that the code produces are shown in Figs. 4 and 5.

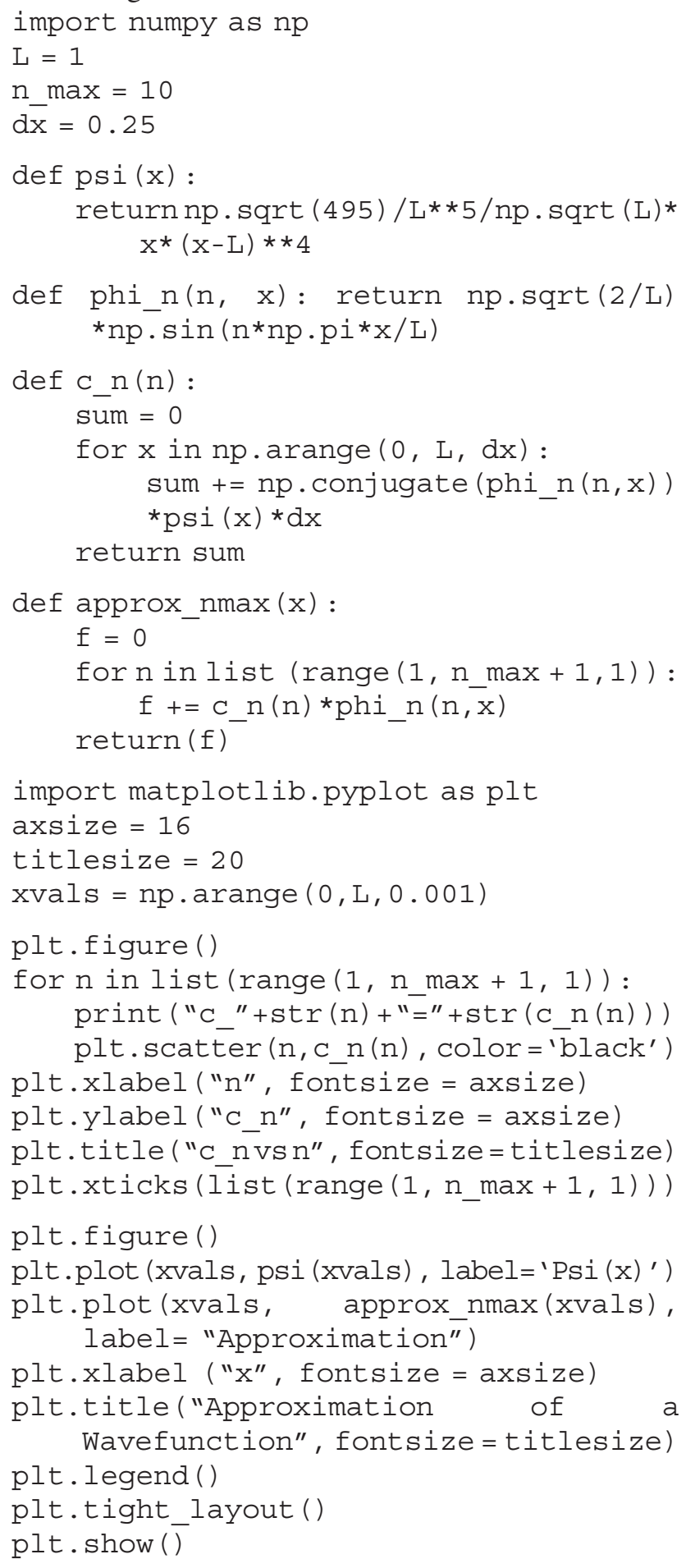




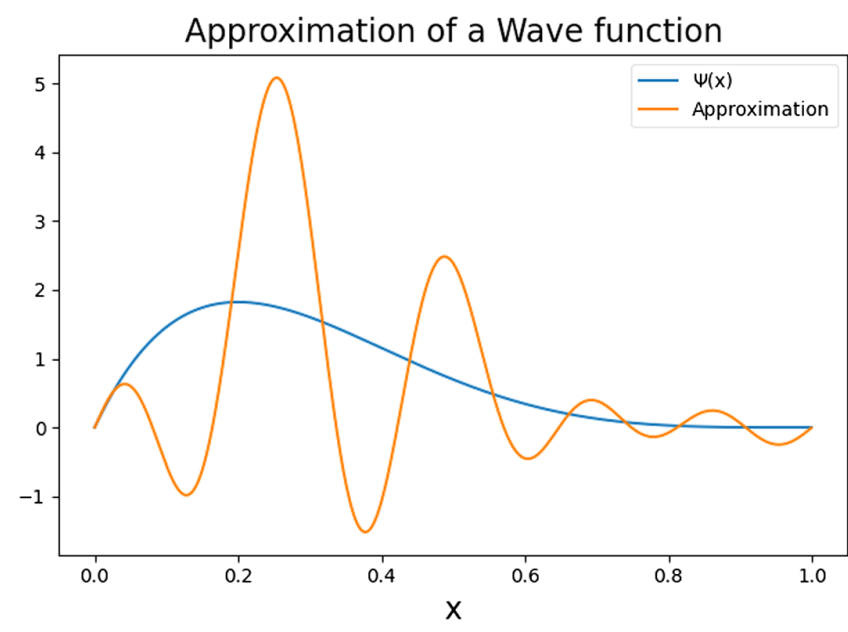

FIG. 4. The wave function of a particle in a box and an approximation up to the first 10 terms in an energy eigenstate of that wave function with probability density on the vertical axis and position on the horizontal axis.

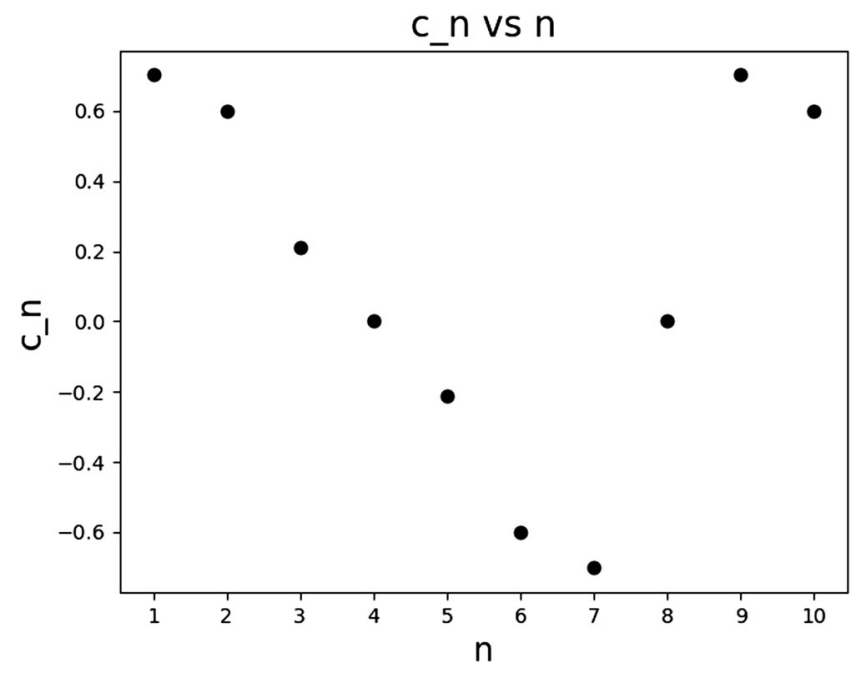

FIG. 5. The inner product of the wave function and an energy eigenstate of a particle in a box, with $c_{n}$ on the vertical axis and the energy level labeled on the horizontal axis.
[1] R. Mirick and S. Wladkowski, Skype in qualitative interviews: Participant and researcher perspectives, Qualitative Rep. 24, 3061 (2019).

[2] L. Gray, G. Wong, G. Rempel, and K. Cook, Expanding qualitative research interviewing strategies: Zoom video communications, Qualitative Rep. 25, 1292 (2020).

[3] M. Sedgwick and J. Spiers, The use of videoconferencing as a medium for the qualitative interview, Int. J. Qualitative Methods 8, 1 (2009).

[4] H. Deakin and K. Wakefield, Skype interviewing: reflections of two PhD researchers, Qualitative Res. 14, 603 (2014).

[5] M. M. Archibald, R. C. Ambagtsheer, M. G. Casey, and M. Lawless, Using zoom videoconferencing for qualitative data collection: Perceptions and experiences of researchers and participants, Int. J. Qualitative Methods 18, 1609406919874596 (2019).

[6] D. R. Johnson, C. P. Scheitle, and E. H. Ecklund, Beyond the in-person interview? How interview quality varies across in-person, telephone, and Skype interviews, Soc. Sci. Comput. Rev. 0894439319893612 (2019), https:// doi.org/10.1177/0894439319893612.

[7] P. Hanna, Using internet technologies (such as Skype) as a research medium: a research note, Qualitative Res. 12, 239 (2012).

[8] J. W. Creswell and J. W. Creswell, Qualitative Inquiry and Research Design: Choosing among Five Approaches, 3rd ed. (SAGE Publications, Los Angeles, 2013).

[9] J. K. Cater, Skype a cost-effective method for qualitative research, Rehabilitation Counselors Educators J. 4, 3 (2011).
[10] S. Weller, Using internet video calls in qualitative (longitudinal) interviews: some implications for rapport, Int. J. Soc. Res. Methodology 20, 613 (2017).

[11] C. A. Manogue and K. S. Krane, Paradigms in physics: Restructuring the upper, Phys. Today 56, No. 9, 53 (2003).

[12] L. Williams, R. R. Kessler, W. Cunningham, and R. Jeffries, Strengthening the case for pair programming, IEEE Softw. 17, 19 (2000).

[13] C. McDowell, B. Hanks, and L. Werner, Experimenting with pair programming in the classroom, in Proceedings of the 8th Annual Conference on Innovation and Technology in Computer Science Education, ITiCSE '03 (Association for Computing Machinery, New York, NY, USA, 2003), pp. 60-64.

[14] C. McDowell, L. Werner, H. Bullock, and J. Fernald, The effects of pair-programming on performance in an introductory programming course, in Proceedings of the 33rd SIGCSE Technical Symposium on Computer Science Education, SIGCSE '02 (Association for Computing Machinery, New York, NY, USA, 2002), pp. 38-42.

[15] L. Williams and R. L. Upchurch, In support of student pairprogramming, ACM SIGCSE Bull. 33, 327 (2001).

[16] S. Weatherford and R. Chabay, Student predictions of functional but incomplete example programs in introductory calculus-based physics, in Proceedings of the 2019 Physics Education Research Conference (2013), pp. 4245, https://www.per-central.org/items/detail.cfm?ID=12934.

[17] M. T. H. Chi, P. J. Feltovich, and R. Glaser, Categorization and representation of physics problems by experts and novices, Cogn. Sci. 5, 121 (1981). 
[18] A. Kimbrough, P. J. Emigh, J. W. Alfson, and E. Gire, Teaching gravitational potential energy: Student interaction with surface manipulatives, in Proceedings of the 2019 Physics Education Research Conference (2020), pp. 288-293, https:// www.compadre.org/per/items/detail.cfm?ID $=15288$.

[19] C. A. Moylan, A. S. Derr, and T. Lindhorst, Increasingly mobile: How new technologies can enhance qualitative research, Qual. Soc. Work 14, 36 (2015).

[20] V. Lo Iacono, P. Symonds, and D. H. Brown, Skype as a tool for qualitative research interviews, Sociol. Res. Online 21, 103 (2016).

[21] X. Wang, S. Hegde, C. Son, B. Keller, A. Smith, and F. Sasangohar, Subjective well-being of Chinese Sina Weibo users in residential lockdown during the COVID-19 pandemic: Machine learning analysis, J. Medical Internet Res. 22, e24775 (2020).

[22] M. É. Czeisler, R. I. Lane, E. Petrosky, J. F. Wiley, A. Christensen, R. Njai, M. D. Weaver, R. Robbins,
E. R. Facer-Childs, L. K. Barger, C. A. Czeisler, M. E. Howard, and S. M. Rajaratnam, Mental health, substance use, and suicidal ideation during the COVID-19 pandemic - United States, June 24-30, 2020, Morbidity Mortality Weekly Rep. 69, 1049 (2020).

[23] J. Xiong, O. Lipsitz, F. Nasri, L. M. W. Lui, H. Gill, L. Phan, D. Chen-Li, M. Iacobucci, R. Ho, A. Majeed, and R. S. McIntyre, Impact of COVID-19 pandemic on mental health in the general population: A systematic review, J. Affective Disord. 277, 55 (2020).

[24] D. A. AlAteeq, S. Aljhani, and D. AlEesa, Perceived stress among students in virtual classrooms during the COVID-19 outbreak in KSA, J. Taibah Univ. Medical Sci. 15, 398 (2020)

[25] M. M. Husky, V. Kovess-Masfety, and J. D. Swendsen, Stress and anxiety among university students in France during Covid-19 mandatory confinement, Compr. Psychiatry 102, 152191 (2020). 